\title{
Inhibition of Dectin-I on Dendritic Cells Prevents Maturation and Prolongs Murine Islet Allograft Survival
}

Ao Ren ${ }^{1-3}$

Zhongqiu Li $\mathbb{D}^{1-3}$

Xuzhi Zhang ${ }^{1-3}$

Ronghai Deng ${ }^{1-3}$

Yi Mal-3

'Organ Transplant Center, The First Affiliated Hospital, Sun Yat-sen University, Guangzhou, People's Republic of China; ${ }^{2}$ Guangdong Provincial Key Laboratory of Organ Donation and Transplant Immunology, The First Affiliated Hospital, Sun Yat-sen University, Guangzhou, People's Republic of China; ${ }^{3}$ Guangdong Provincial International Cooperation Base of Science and Technology (Organ Transplantation), The First Affiliated Hospital, Sun Yat-sen University, Guangzhou, People's Republic of China

Correspondence: Ronghai Deng; Yi Ma Organ Transplant Center, The First Affiliated Hospital, Sun Yat-sen university, No. 58 Zhongshan Er Road, Guangzhou 510080, People's Republic of China $\mathrm{Tel} / \mathrm{Fax}+86-20-87306082$ Email mddrh8I@I63.com; anhuimayi2002@I63.com
This article was published in the following Dove Press journal: Journal of Inflammation Research

Introduction: The ability of dendritic cells (DCs) to initiate an immune response or induce immune tolerance depends on their maturation status. Dendritic-cell-associated C-type lectin 1 (Dectin-1) plays a key role in the differentiation, activation, and maturation of DCs. Therefore, we hypothesized that inhibition of Dectin-1 could prevent DC maturation and induce immune tolerance of transplanted organs.

Methods: DCs were transduced with a recombinant lentiviral vector to inhibit Dectin-1 and then were injected into a murine recipient before islet transplantation. C57BL/6 mice $(\mathrm{H}-2 \mathrm{~b})$ were treated with lentiviral vector-Dectin-1-RNAi-DC (DC-Dectin-1-RNAi group), lentiviral vector-GFP DCs (DC-GFP group), and PBS (control group). Pancreatic islet transplantation was performed and graft survival was recorded. The proportions of regulatory $\mathrm{T}$ cells, Th1 cells, and Th17 cells in the spleen and draining lymph nodes, and serum levels of interleukin (IL)-10, IL-17, and interferon (INF)- $\gamma$ were measured.

Results: The inhibition of Dectin-1 resulted in low expression of MHC-II and costimulatory molecules in DCs. Murine recipients treated with DC-Dectin-1-RNAi had longer islet allograft survival time, a reduction in the levels of Th1 and Th17 cells and secreted cytokines, and an increase of Treg cells.

Conclusion: The inhibition of Dectin-1 by recombinant lentiviral vector Dectin-1-RNAi inhibits the maturation and activation of DCs, affects the differentiation of T cell subsets, and prolongs allograft survival.

Keywords: dendritic cell, Dectin-1, immune tolerance, islet transplant, lentiviral vector

\section{Introduction}

Type 1 diabetes is characterized by the progressive destruction of pancreatic $\beta$ cells. Pancreatic islet transplantation has been shown to improve glycemic control in patients with type 1 diabetes. ${ }^{1-3}$ However, rejection of islet allografts is a major limitation of the procedure. ${ }^{4}$ As such, developing new and effective methods to induce immune tolerance and improve graft survival is an important area of research.

Dendritic cells (DCs) are key mediators of immunity and immune tolerance, and provide a link between innate and adaptive immunity. ${ }^{5}$ DCs capture antigens such that they can be processed by major histocompatibility complex (MHC) on the cell surface, which thus stimulates the initiation of an effective adaptive immune response. A number of studies have shown that immune tolerance is induced by immature, tolerogenic DCs (tolDCs). ${ }^{6}$ These cells express low levels of $\mathrm{T}$ cell 
costimulatory molecules and reduce the secretion of proinflammatory cytokines, thereby inducing immunological tolerance. $^{6}$

Dendritic-cell-associated C-type lectin 1 (Dectin-1) is a C-type lectin receptor (CLR), that is primarily expressed on myeloid cells, macrophages, neutrophils, and DCs. ${ }^{7}$ Dectin-1 has been primarily studied in the context of anti-fungal immunity. Recently, Dectin-1 has been considered to play a role in promoting the inflammatory diseases through immune response. ${ }^{8-10}$ Dectin-1 promotes DC maturation and triggers a potent anti-tumor immune response as well as protective anti-fungal immunity. ${ }^{11}$ Activation of Dectin-1 increases the production of cytokines and the T cell stimulatory capacity of DCs. ${ }^{12}$

Based on the aforementioned studies and the positive effect of Dectin-1 with respect to an immune response, we hypothesized that inhibition of Dectin-1 will inhibit maturation of DCs and induce immune tolerance. We tested this hypothesis by transducing DCs with a lentiviral vector (LV) Dectin-1-RNAi to inhibit Dectin-1. We then used a murine model to determine if DC-Dectin-1-RNAi prolonged pancreatic islet allograft survival.

\section{Materials and Methods}

\section{Animals}

Male C57BL/6 mice (H-2 ${ }^{\text {b }}$ ( 8 to 10 weeks old, SPF grade) and male BALB/c mice $\left(\mathrm{H}-2^{\mathrm{d}}\right)$ ( 8 to 10 weeks old, SPF grade) were purchased from the Experiment Animal Center of Sun Yat-sen University (Guangzhou, China). All animal experiments were performed according to guidelines established by Animal Care and Use Committee of Sun Yat-sen University. And this experiment was approved by the Ethics Committee of The First Affiliated Hospital of Sun Yat-sen University (Approval No. (2020) 287). C57BL/6 mice were used as transplant recipients, and $\mathrm{BALB} / \mathrm{c}$ mice were used as transplant donors.

\section{Reagents}

Recombinant murine granulocyte/macrophage colonystimulating factor (GM-CSF) and interleukin (IL)-4 were purchased from PeproTech (NJ, USA). Rat antimouse fluorescence-conjugated CD3, CD4, CD8, CD25, Foxp3, interferon (IFN)- $\gamma$, IL-17, CD11c, CD40, CD80, CD86, CD83, and MHC-II (I-A/I-E), as well as their corresponding isotype controls, were purchased from BD Pharmingen ${ }^{\mathrm{TM}}$ (San Diego, CA, USA). Microbeadconjugated anti-CD11c and LS separation columns were purchased from Miltenyi Biotec (Bergisch Gladbach,
Germany). Anti-mouse insulin and glucagon monoclonal antibodies (mAbs) were purchased from Cell Signaling Technology (Danvers, US). The lentiviral short hairpin RNA vector (shRNA) Dectin-1-RNAi-GFP was constructed by Shanghai Genechem Co., Ltd. (Shanghai, China). Enzyme-linked immunosorbent assay (ELISA) kits for measurement of INF- $\gamma$, IL-10, and IL-17 were purchased from CUSABIO (Wuhan, China), and a kit for measuring tumor growth factor (TGF)-b was purchased from eBioscience. Streptozotocin (STZ), lipopolysaccharide (LPS), and Histopaque1077 were purchased from Sigma-Aldrich (St. Louis, CA, USA). Diphenylthiocarbazone (DTZ), acridine orange (AO), propidium iodide (PI), Liberase TL, a blood glucose meter, and blood glucose test strips were purchased from Roche (Basel, Switzerland).

\section{Generation of Bone Marrow Derived Dendritic Cells (BMDCs)}

The femurs and tibias of healthy $\mathrm{C} 57 \mathrm{BL} / 6$ mice were obtained via a sterile procedure after the mice were killed. The bone cavities were flushed with PBS, and the red blood cells (RBCs) were lysed. Bone marrow cells were resuspended in RPMI 1640 medium at a concentration of $0.5-1 \times 10^{6}$ cells $/ \mathrm{mL}$, then cultured in 6 -well plates with complete culture medium (RPMI 1640 supplemented with $10 \% \mathrm{FBS}, 1 \%$ penicillin-streptomycin, $20 \mathrm{ng} / \mathrm{mL}$ recombinant murine GM-CSF, and $10 \mathrm{ng} / \mathrm{mL}$ IL-4) for 6 days to obtain immature DCs (imDCs). The imDCs were then purified by immunomagnetic selection of $\mathrm{CD} 11 \mathrm{c}+$ cells with anti-CD11c-conjugated microbeads.

\section{LV-Dectin-I-RNAi-GFP Transduction of DCs}

Purified imDCs were transduced with LV-Dectin-1-RNAiGFP (DC-Dectin-1-RNAi) or LV-GFP (DC-GFP) at a multiplicity of infection (MOI) of 1:100 for $72 \mathrm{~h}$ in serum-free RPMI 1640. Cells were stimulated with LPS $(1 \mu \mathrm{g} / \mathrm{ml})$ for 48 $\mathrm{h}$ to become mature DCs (mDCs). The expressions of CD40, CD80, CD83, CD86, and MHC-II were detected by flow cytometry to determine cell maturation. Recipient mice were injected vis the tail vein with a total of $2 \times 10^{6}$ DC-Dectin-1RNAi or DC-GFP cells 1 day prior to islet transplantation.

\section{Western Blotting}

Proteins were extracted with a lysis buffer (Roche, Switzerland) containing $1 \%$ protease inhibitor, and $10 \%$ 
phosphatase inhibitor. Protein concentration was measured using a bicinchoninic acid (BCA) protein assay kit (Beyotime Institute of Biotechnology, Shanghai, China) according to the manufacturer's instructions. Equal amounts of protein were separated by $12 \%$ sodium dodecyl sulfate polyacrylamide gel electrophoresis (SDSPAGE) and subsequently transferred to nitrocellulose membranes. The membranes were incubated with antiDectin-1 (\#ab140039; Abcam, Cambridge, UK, 1:1000) and anti- $\beta$-actin (\#2060; Dia-an Biotechnology, Wuhan, China, 1:1000) overnight at $4^{\circ} \mathrm{C}$. After washing, the membrane was incubated with a horseradish peroxidase-conjugated (HRP)-goat anti-mouse IgG (Q1001; Dia-an Biotechnology, 1:2000) and HRP-goat anti-rabbit IgG (Q1002; Dia-an Biotechnology, 1:2000) secondary antibody for $1 \mathrm{~h}$.

\section{RNA Extraction and Quantitative RT-PCR (qRT-PCR)}

RNA was extracted from DCs using TRIZOL (Invitrogen, Carlsbad, CA, USA) according to the manufacturer's protocol. The concentration and quality of the RNA were detected via a Nanodrop 2000 (Thermo Fisher Scientific, Waltham, MA, USA). RNA was reverse transcribed into singlestranded cDNA using a PrimerScript ${ }^{\mathrm{TM}} \mathrm{RT}$ reagent kit with gDNA Eraser (TaKaRa, Japan) according to the manufacturer's instructions. The relative amount of mRNA was calculated using the $2^{-\Delta \Delta \mathrm{Ct}}$ method and normalized to the mean expression of GAPDH. Each experiment was repeated at least 3 times. Primers are shown in Table 1.

\section{Mixed Leukocyte Reaction (MLR) Analysis}

The stimulatory capacity of DCs was tested by mixed leukocyte reaction (MLR) analysis. DCs pretreated with mitomycin C (25 $\mu \mathrm{g} / \mathrm{mL}$, Amresco, USA) were co-cultured with splenic naive $\mathrm{T}$ cells from Balb/c mice at DC: T-cell ratios of $10: 1,20: 1$ and $40: 1$ for $72 \mathrm{~h}$ in RPMI

Table I Sequences of Primers Used for Real-Time Quantitative Polymerase Chain Reaction

\begin{tabular}{|l|l|}
\hline Gene & Sequences $\left(\mathbf{5}^{\prime} \rightarrow \mathbf{3}^{\prime}\right)$ \\
\hline GAPDH & $\begin{array}{l}\text { S-CCTCGTCCCGTAGACAAAATG } \\
\text { A-TGAGGTCAATGAAGGGGTCGT }\end{array}$ \\
\hline Dectin-I & $\begin{array}{l}\text { F- ACTGAAGATAGACAACTCAAAAGAA } \\
\text { R- TCCAAAATGCATTAATACGGTGAGA }\end{array}$ \\
\hline
\end{tabular}

1640 with $10 \% \mathrm{FBS}$ at $37^{\circ} \mathrm{C}$. The total volume was adjusted to $200 \mu \mathrm{L} /$ well. Then, Cell Counting Kit (CCK-8; Tongren, Japan) was added to the medium for $4 \mathrm{~h}$ before the end of culture, and $\mathrm{T}$ cell group (without dendritic cells incubated together) and only RPMI 1640 with 10\% FBS medium group were established as negative control group and background group. Absorbance (A) at a wavelength of $450 \mathrm{~nm}$ was measured using a Microplate reader (Bio-Rad, iMark $^{\mathrm{TM}}$ ). The results were expressed as the stimulation index (SI), calculated by the following formula: stimulation index $=$ (absorbance of the sample - the background absorbance)/(absorbance of the negative control - the background absorbance).

To evaluate secretion of cytokines (IL-10, IL-17, IFN$\gamma$ ) by DCs, the culture supernatants were collected after 72 $\mathrm{h}$ and cytokine concentrations were measured using ELISA kits according to the manufacturer's protocols.

\section{Phagocytosis Assay}

To investigate the phagocytosis ability of DCs, mannose receptor-mediated endocytosis was determined by measuring the cellular uptake of TRITC-dextran. Approximately $1 \times 10^{6}$ DCs were resuspended in $250 \mu \mathrm{L}$ of media containing $25 \mu \mathrm{g} / \mathrm{mL}$ TRITC-dextran. After incubation with TRITC-dextran at $37^{\circ} \mathrm{C}$ or $4^{\circ} \mathrm{C}$ for $2 \mathrm{~h}$, DCs were washed 3 times with $4^{\circ} \mathrm{C}$ PBS and $1 \% \mathrm{FBS}$, then analyzed by fluorescence-activated single-cell sorting (FACS).

\section{Pancreatic Islet Transplantation}

Islet transplantation was performed as previously described. ${ }^{13}$ Briefly, streptozotocin $(200 \mathrm{mg} / \mathrm{kg}$ ) was used to induce diabetes in $\mathrm{C} 57 \mathrm{BL} / 6$ mice. Islet transplantation was performed when the recipient blood glucose level was $\geq 16.7 \mathrm{mmol} / \mathrm{L}$ for 3 consecutive days. Pancreatic islets were isolated from $\mathrm{BALB} / \mathrm{c}$ mice. After clamping the duodenal ampulla, the pancreatic duct was cannulated and the pancreas perfused with Liberase TL. The pancreas was then harvested and stored on ice. Islets were isolated from BALB/c mice via reverse perfusion of the common bile duct with Liberase TL and purified by density gradient separation (Histopaque 1077). Isolated islets $(\mathrm{n}=350)$ were transplanted under the kidney capsule (superior pole) of the recipient using a glass capillary tube probe. If the blood glucose level $<200 \mathrm{mg} / \mathrm{dL}$, the transplantation was considered successful, rejection of islet allografts was defined as a blood glucose level $>16.7 \mathrm{mmol} / \mathrm{L}$ (300 mg/dL) for at least 2 consecutive days. 


\section{Intraperitoneal Glucose Tolerance Test}

Intraperitoneal glucose tolerance tests (IPGTTs) were performed short term (day 7-14 after transplantation) and long term (day 25-30 after transplantation), and the isletbearing kidneys were removed at these time points. Briefly, mice were injected intraperitoneally with a glucose solution ( $2 \mathrm{~g} / \mathrm{kg})$ after $4-6 \mathrm{~h}$ of fasting, and glucose levels in tail tip blood were measured every $15 \mathrm{~min}$ for $120 \mathrm{~min}$. The IPGTT was repeated after removal of islet-bearing kidney to confirm that the recipient blood glucose response was due to the bioactivity of the donor islet allograft, rather than recovery of the recipient STZ-damaged pancreas.

\section{Flow Cytometry Analysis}

Lymphocyte cells were collected from the recipient spleen and draining lymph nodes on day 7 after transplantation, and on the day rejection was identified, and stained with the previously indicated antibodies at $4^{\circ} \mathrm{C}$ for $30 \mathrm{~min}$, and analyzed with a flow cytometer (BD FACS Calibur, US). Th1, Th17, Treg, and intracellular cytokine staining analyses were performed following stimulation with Cell Stimulation Cocktail plus Protein Transport Inhibitor for 4-6 h. The cells were fixed and permeabilized using a Foxp3/Transcription Factor Staining Buffer Set, then stained for intracellular cytokines. Data were analyzed with FlowJo software. Th1 cells were defined as $\mathrm{CD}^{+} /$ $\mathrm{CD} 8^{-} / \mathrm{IFN}^{+}$, Th17 cells were defined as $\mathrm{CD}^{+} / \mathrm{CD}^{+} / \mathrm{IL}^{-}$ $17^{+}$, and Treg cells were defined as $\mathrm{CD} 4^{+} / \mathrm{CD} 25^{+} /$Foxp $^{+}$.

\section{ELISA}

ELISA testing for serum cytokine levels were performed as previously described. ${ }^{14}$ Briefly, blood were collected from the retro-orbital sinus on day 7 after transplantation, and on the day rejection was identified post-transplantation. Serum samples were prepared, and the levels of IL10 , IL-17, and INF- $\gamma$ were measured by ELISA conducted according to manufacturer's instructions.

\section{Immunohistochemistry and Hematoxylin and Eosin (HE) Analysis}

Graft tissues were harvested on days 7 and 25 after transplantation, fixed with $10 \%$ paraformaldehyde, embedded in paraffin, cut into 3-5 $\mu \mathrm{m}$ thick slices, and stained with HE or with anti-mouse insulin or glucagon monoclonal antibodies according to the manufacturer protocols.

\section{Statistical Analyses}

Data were expressed as mean \pm standard deviation. Differences between groups were examined by ANOVA. Graft survival was analyzed using Kaplan-Meier survival curves compared with the Log rank test. All statistical analyses were performed with SPSS version 19.0 software. Values of $\mathrm{P}<0.05$ were considered statistically significant.

\section{Results \\ Genetically Modified Lentiviral Vector Increases the Transduction Efficiency of DCs}

The lentiviral vector of shRNA Dectin-1-RNAi-GFP was purchased from Shanghai Genechem Co., Ltd., and was modified to increase the transduction efficiency of DCs. ${ }^{15}$ The DCs were purified to $>90 \%$ by immunomagnetic selection of $\mathrm{CD} 11 \mathrm{c}+$ cells with anti-CD11c-conjugated microbeads (Figure 1F). Purified DCs were transduced with LV-Dectin-1-RNAi-GFP or LV-GFP at an MOI of 1:100 for $72 \mathrm{~h}$ in serum-free RPMI 1640. GFP fluorescence was detected by a fluorescent microscope (Olympus IX51, Japan) after 48-72 h (Figure 1A and B). Green fluorescence indicated successful infection of DCs with the LV-Dectin-1-RNAi-GFP or LV-GFP (Figure 1B). GFP fluorescence could be observed until spontaneous apoptosis of the mDCs. To determine the transduction efficiency of DCs, Dectin-1 mRNA and protein expression levels were measured. The results of qRT-PCR indicated that Dectin-1 mRNA expression was significantly decreased in DC-Dectin-1-RNAi cells (Figure 1E). This result was verified by Western blot analysis which showed that Dectin-1 protein levels were also decreased in DCDectin-1-RNAi cells (Figure 1C and D). These results indicated that the lentiviral vector effectively transferred the Dectin-1-RNAi gene into DCs.

\section{DC-Dectin-I-RNAi Decreases the Expression of MHC and Costimulatory Molecules}

DC maturation can be stimulated by LPS, which acts through pattern recognition receptors on DCs (such as Dectin-1). ${ }^{16}$ To determine the effect of inhibition of Dectin-1 on DC maturation purified imDCs, DC-GFP, and DC-Dectin-1-RNAi cells were stimulated with LPS $(1 \mu \mathrm{g} / \mathrm{mL})$ for $48 \mathrm{~h}$. DCs were collected and stained with fluorescent-conjugated CD40, CD80, CD83, CD86, and 
A

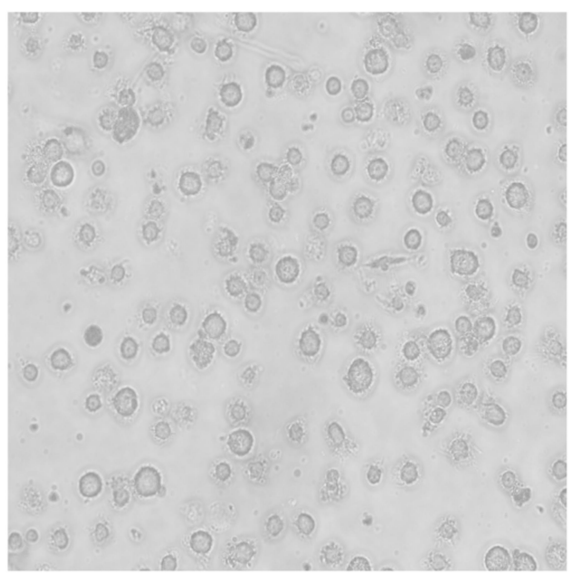

C

\section{Control DC-Dectin-1-RNAi}
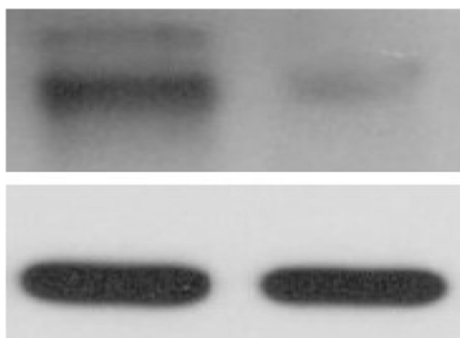

$\beta$-actin

Dectin-1

$\beta$-actin

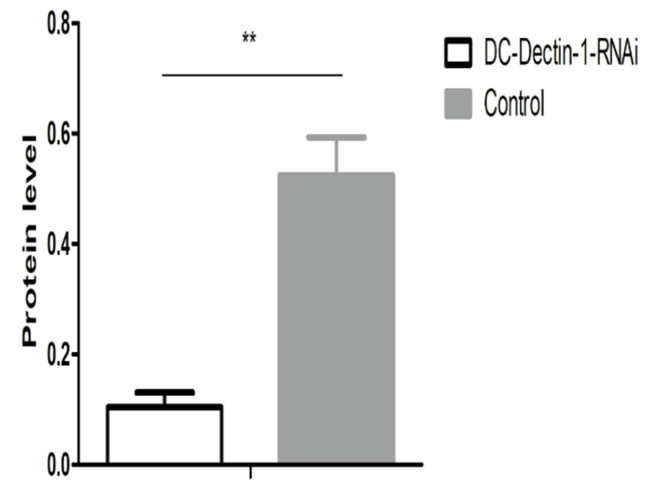

E

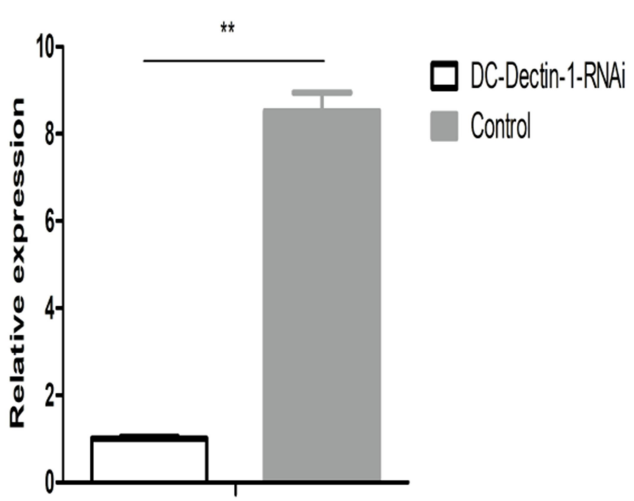

D

\section{B}

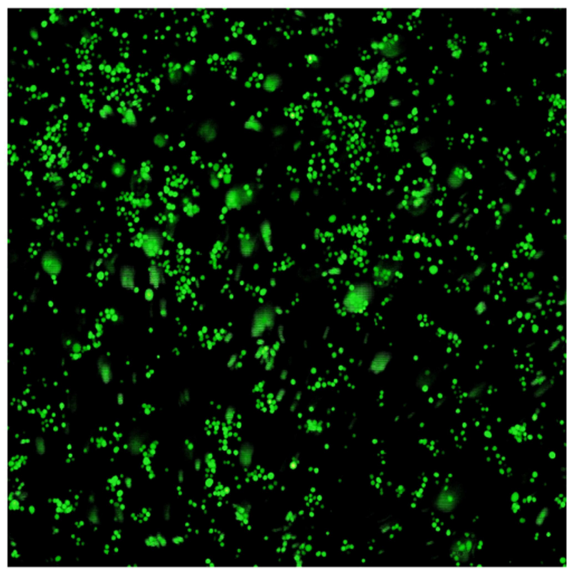

$\mathbf{F}$

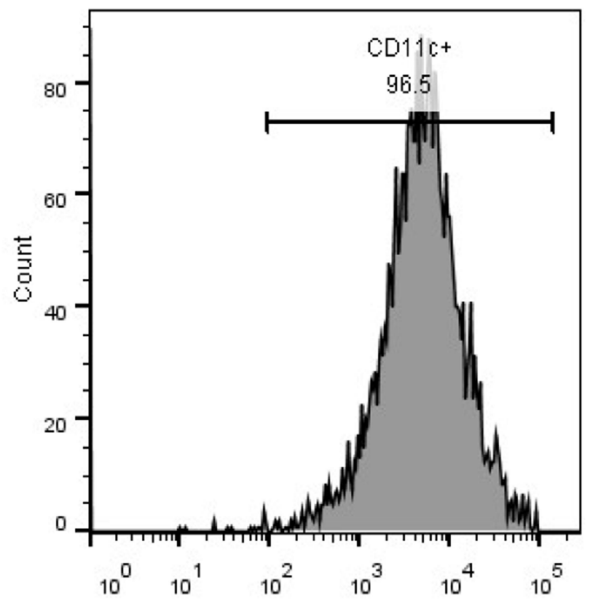

Figure I DCs transduced with LV-Dectin-I-RNAi-GFP. (A) mDCs purified with CDIlc microbeads. *( $\times 400)$ (B) The expression of GFP in DCs $72 \mathrm{~h}$ after LV-Dectin-IRNAi-GFP transduction observed in fluorescence microscopy. $(\times 200)($ C and D) Western blot analysis of Dectin-I protein expression in DCs transduced with LV-Dectin-IRNAi-GFP. $n=3$. (E) qRT-PCR analysis of Dectin-I mRNA expressions in DCs transduced with LV-Dectin-I-RNAi-GFP. GADPH served as an internal control. (F) Purity of CDIIc+ DCs. Data represent the mean \pm SD. **P $<0.01$. 
MHC-II mAbs. The DC-Dectin-1-RNAi group exhibited lower expression levels of CD40, CD80, CD83, CD86, and MHC-II than the DC-GFP group (Figure 2). Results of the MLR showed that the level of $\mathrm{T}$ cell proliferation induced by DCs. DC-GFP had a stronger stimulatory capacity than DC-Dectin-1-RNAi (Figure 2B), and the difference was significant, especially when DC and T cell ratio was $1: 10$. The supernatant of the MLR (DC: $\mathrm{T}$ cell $=$ 1:10) was collected for analysis by ELISA; in the DCDectin-1-RNAi group IL-17 and IFN- $\gamma$ levels were decreased and the level of IL-10 was increased (Figure 2C). Results of phagocytosis assay suggested that DC-Dectin-1-RNAi group showed stronger phagocytic ability than DC-GFP group (Figure 2D). Taken together, these results indicated that inhibition of Dectin-1 could effectively block the antigen-presenting and $\mathrm{T}$ cell activating capacity of DCs by inhibiting expression of costimulatory molecules and $\mathrm{MHC}$.

\section{DC-Dectin-I-RNAi Protects Allograft Function and Prolongs Graft Survival}

To examine the influence of DC-Dectin-1-RNAi on transplantation immunity in vivo, a murine model of islet allograft transplantation with diabetic mice was used. After isolation, the purity of islets was evaluated by DTZ staining and activity was evaluated by AO-PI staining. The results showed that the purity and activity of the islets were high (Figure 3A). To investigate the functional activity of transplanted islet allografts in vivo, an IPGTT (2 $\mathrm{g} / \mathrm{kg})$ was performed on day 7 and 25 to assess glucose tolerance after 4-6 h of fasting, and glucose levels in tail tip blood were measured every $15 \mathrm{~min}$ for $120 \mathrm{~min}$ (Figure 3B and C). Baseline blood glucose levels were similar in all groups before the IPGITT. After glucose injection, the blood glucose level increased sharply, peaked at $15 \mathrm{~min}$, and then decreased over time. However, there was a greater and swifter response to the glucose boost in the DC-Dectin-1-RNAi group than in the other groups on day 7 ( $p<0.0001$ ) (Figure $3 \mathrm{~B}$ ). On day 25, the IPGTT was repeated after removal of the islet-transplanted kidney to verify that the blood glucose response of the recipient was islet allograft-dependent $(p<0.0001)$ (Figure 3C). The islet-transplanted kidney was removed and stained with $\mathrm{HE}$ and for insulin and glucagon to confirm the presence of islet grafts (Figure 3E). After islet transplantation, non-fasting blood glucose levels were monitored daily. DC-Dectin-1-RNAi significantly prolonged the survival time of the islet allografts as compared to the other 2 groups (both, $\mathrm{P}<0.01$ ) (Figure 3D).

\section{DC-Dectin-I-RNAi Reduced the Numbers of ThI and ThI7 Cells and Levels of Secreted Cytokines, and Increased the Number of Treg Cells}

To evaluate potential mechanism by which DC-Dectin-1RNAi prolonged islet allograft survival in diabetic mice, changes in $\mathrm{T}$ cell subsets and their secreted cytokines were measured (Figures 4 and 5). Lymphocytes were collected from recipient spleens and draining lymph nodes on day 7 and on the day rejection was identified post-transplantation. The lymphocytes were stained with fluorescent mAbs and analyzed by flow cytometry. The results indicated that on day 7 the DC-Dectin-1-RNAi group exhibited significantly reduced proportions of Th1 cells and Th17 cells in both draining lymph nodes and splenocytes as compared to the control group and the DC-GFP group (all, $\mathrm{P}<0.05$ ) (Figure 4A and B). The DC-Dectin-1-RNAi group also exhibited reduced proportion of Th1 and Th17 cells in splenocytes on day of rejection. In addition, the proportions of Treg cells in both draining lymph nodes and splenocytes were significantly different between the DC-Dectin-1-RNAi group and the other 2 groups on day 7 (all, $\mathrm{P}<0.05$ ) (Figure 4A and B).

Serum levels of IL-10, IL-17, and INF- $\gamma$ are summarized in Figure 5. At day 7 post-transplantation, INF- $\gamma$ and IL-17 levels were significantly lower (both, $\mathrm{P}<0.01$ ) and IL-10 was significantly higher $(\mathrm{P}<0.05)$ in the DCDectin-1-RNAi group compared to the DC-GFP group. On the day of rejection post-transplantation, the INF- $\gamma$ was significantly lower in the DC-Dectin-1-RNAi group compared to the other 2 groups (both, $\mathrm{P}<0.01$ ).

\section{Discussion}

This study demonstrated that DC-Dectin-1-RNAi prolonged islet allograft survival, and was associated with reduced levels of costimulatory molecules and cytokines, reduced numbers of Th1 and Th17 cells, and increased numbers of Treg cells. A genetically modified lentiviral vectors was used to enhance the transduction efficiency of DCs, ${ }^{17}$ and the results showed that DCs exhibited a high expression of GFP indicating the high transduction efficiency. 
A

CD40

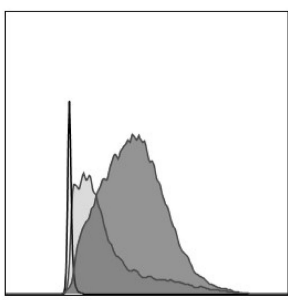

CD80

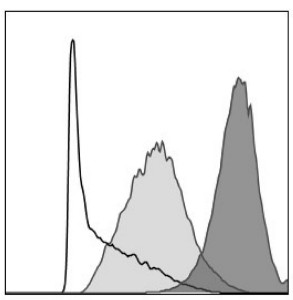

Control

CD83

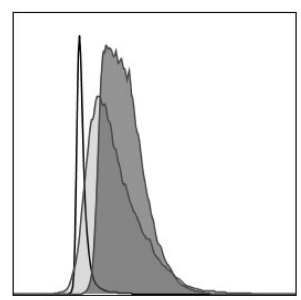

DC-GFP

CD86

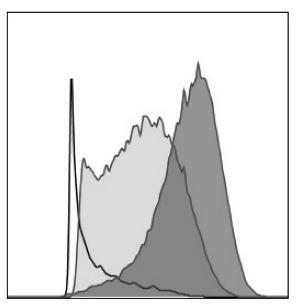

DC-Dectin-1-RNAi

MHC-II

B

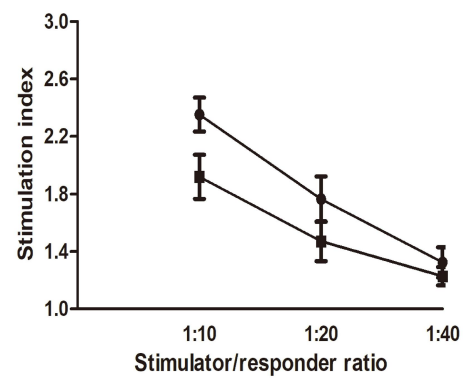

- DC-GFP

- DC-Dectin-1-RNAi

C
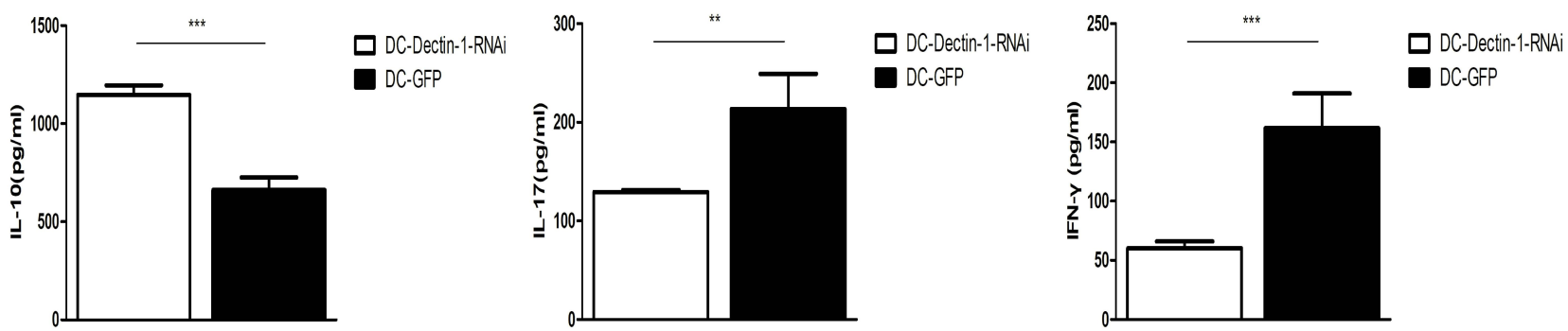

D

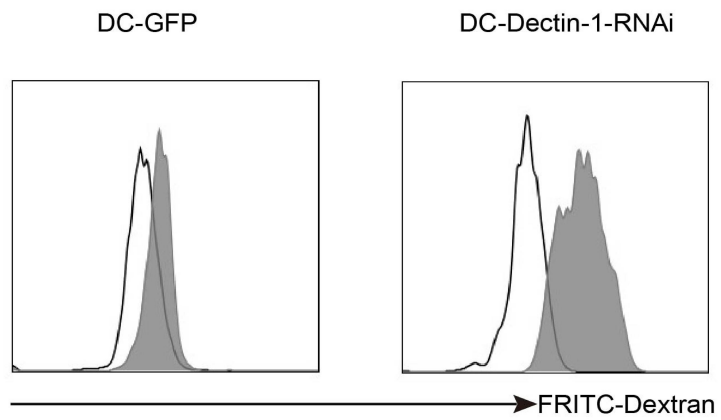

$\square 7^{\circ}$

$\square 4^{\circ}$
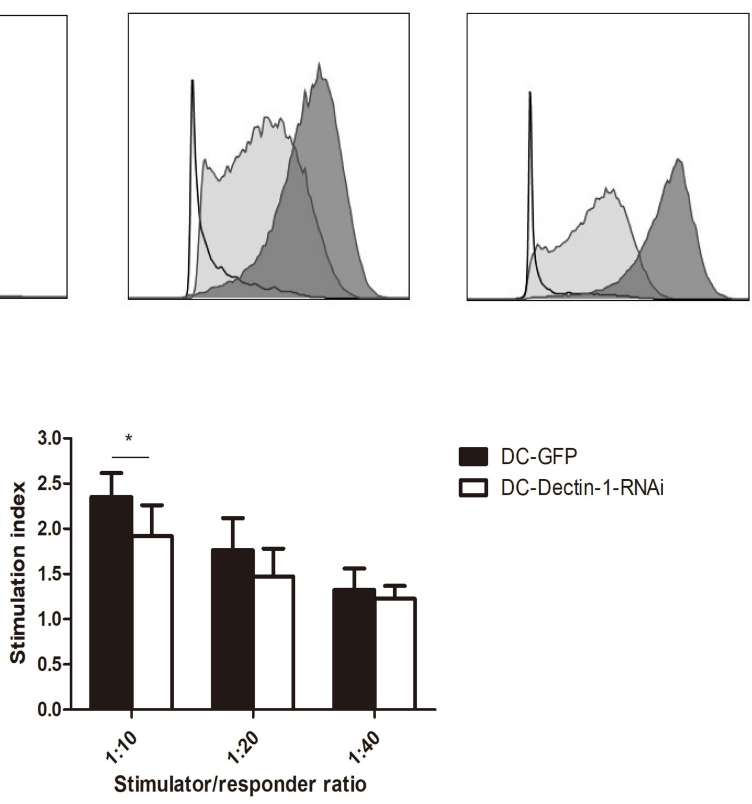

DC-GFP

DC-Dectin-1-RNAi 

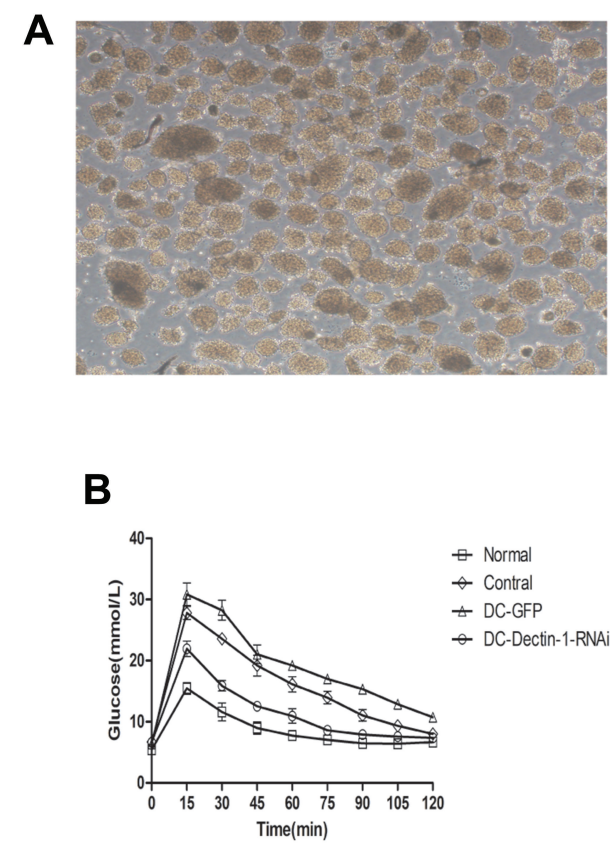

B

E
C

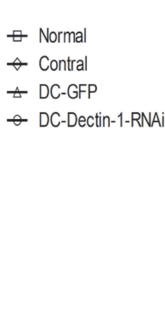

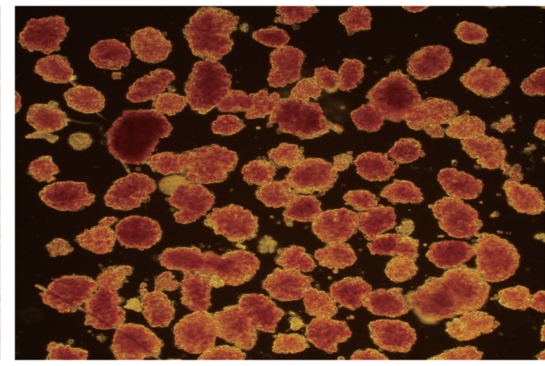

DTZ

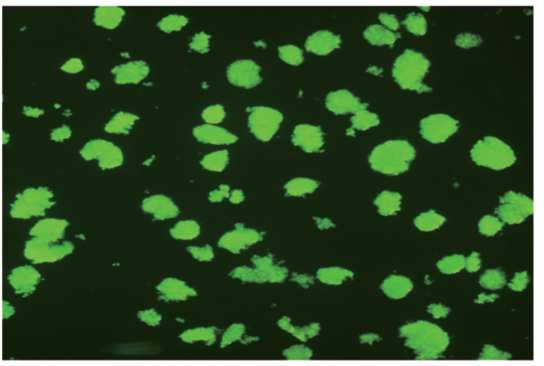

A0-PI

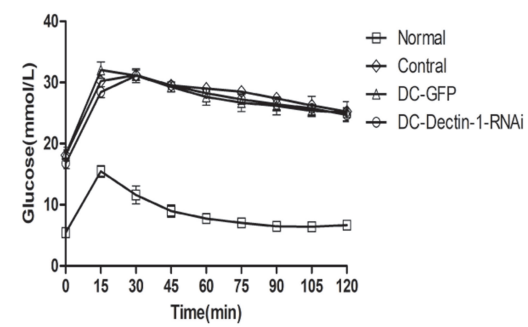

D

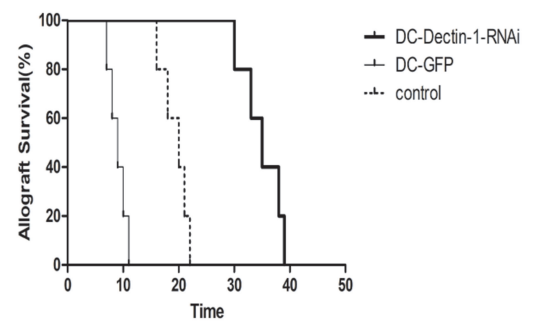

HE

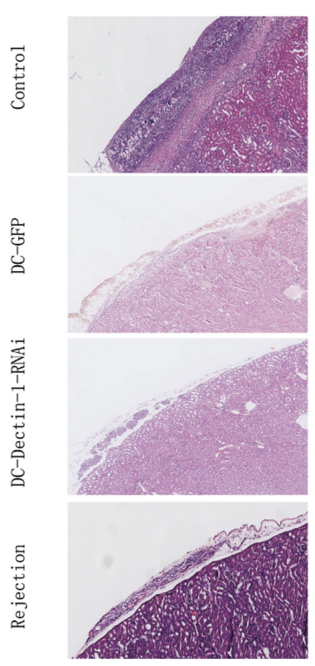

Insulin

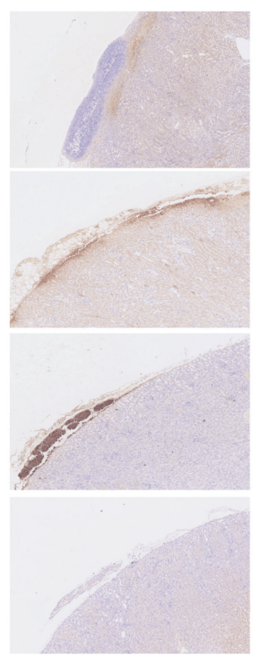

Glucogan

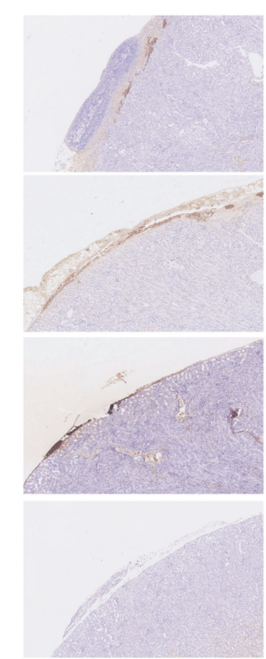

Figure 3 DCs transduced with LV-Dectin-I-RNAi-GFP prolonged the islet allografts survival. (A) Purified islets isolated from the perfusion, digestion, and purification procedure. The purity of islets was evaluated by DTZ staining and the activity was evaluated by AO-PI staining $(\times 40)$. (B) Intraperitoneal glucose tolerance tests (IPGTTs) were performed on day 7 post-transplantation to test recipient glucose tolerance after $4-6 \mathrm{~h}$ of fasting. (C) IPGTTs were repeated after the removal of islet-bearing kidney to confirm recipient blood glucose response was islet allograft dependent. (The upper limit of the blood glucose meter is $33.3 \mathrm{mmol} / \mathrm{L}$, and if the blood glucose level was above the limit it was recorded as $33.3 \mathrm{mmol} / \mathrm{L}$. Data are expressed as mean only.). (D) Survival curve of islet grafts. Graft survival between groups of PBS control, DC-GFP, and DC-Dectin-I-RNAi was compared with Kaplan-Meier analysis ( $n=5$ animals/group). (E) On day 7 and rejection after transplantation, kidneys were removed and stained with the indicated markers. DC-Dectin-I-RNAi treated recipients displayed higher expression of insulin and glucagon compared to the other groups, indicating greater function and survival of islet grafts $(\times 200)$.

primarily expressed on CD11b+ DCs, and study has shown the number of CD11b+ DCs in lymph nodes is significantly reduced in Dectin-1-/- mice compared with wildtype mice. ${ }^{21}$ It has also been reported that Dectin-1 plays an essential role in mediating the polysaccharide-induced activation of DCs resulting in maturation of DCs and subsequent activation of the adaptive immune response. ${ }^{22}$ Takagawa et al ${ }^{8}$ showed that an increase of colitis severity was associated with lamina propria DCs that were associated with increased Dectin-1-induced NF- $\mathrm{kB}$ activation and proinflammatory cytokine secretion. In order to better understand the role of Dectin-1 in stimulating DC maturation, we studied the consequences of Dectin-1 inhibition in DCs using a genetically modified lentiviral vector. Our results revealed that inhibition of Dectin-1 resulted in inhibition of DC maturation, which was characterized by 
A
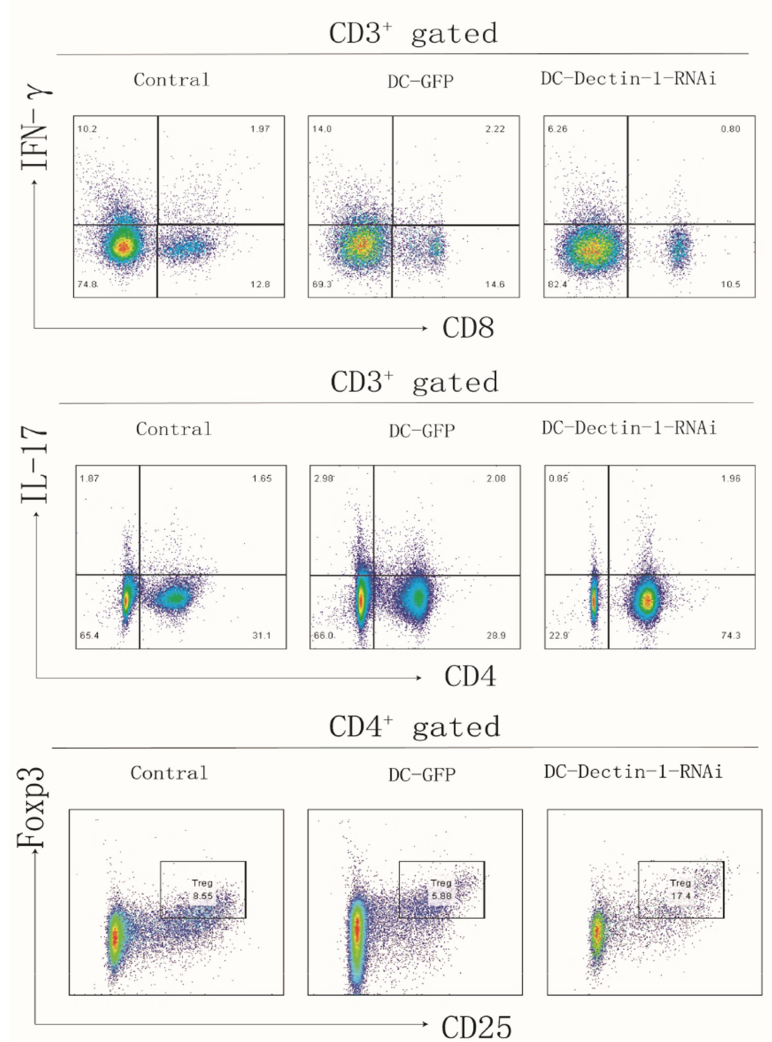

B
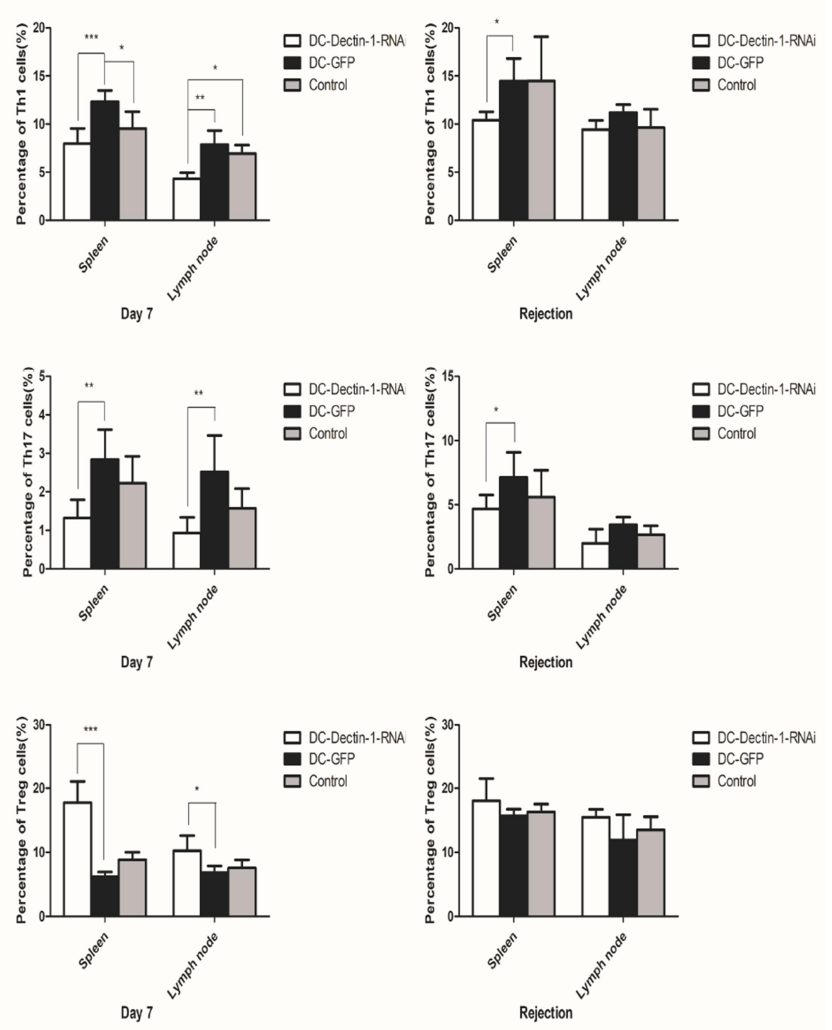

Figure 4 DC-Dectin- I-RNAi reduced the population of ThI and Th I7 cells, increased the frequency of Treg cells (A and B). The percentage of ThI, Th I7, and Treg in draining lymph nodes and spleens were analyzed after transplantation (A only shows day 7 in spleens). Data represent the mean $\pm S D$. $* P<0.05, * * P<0.01, * * * P<0.0001, n=5$ animals/ group.
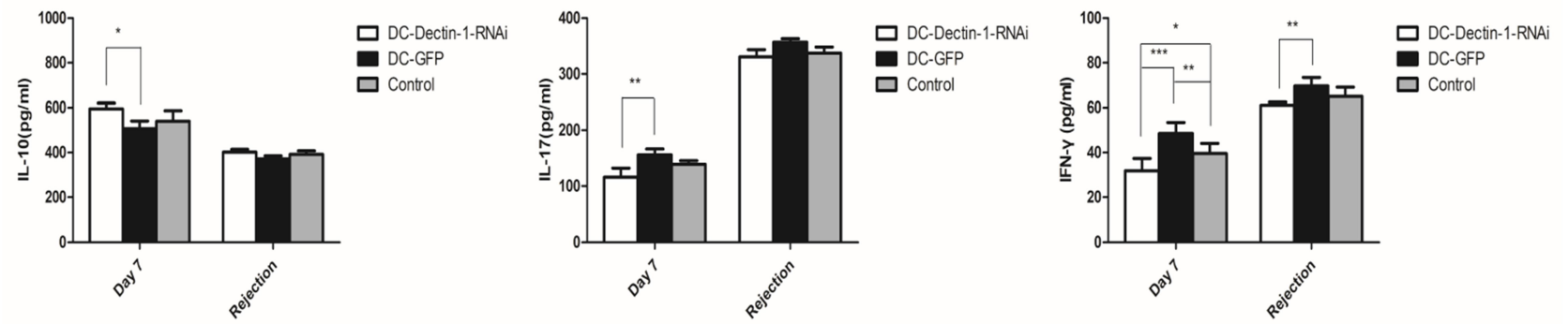

Figure 5 Cytokines level of serum were analyzed on day 7 after transplantation and on the day of rejection, DC-Dectin-I-RNAi reduced the IL-I 7 and IFN- $\gamma$ level of serum, increased the IL- 10 level of serum. Data represent the mean $\pm \mathrm{SD}$. $* \mathrm{P}<0.05, * * \mathrm{P}<0.01, * * * \mathrm{P}<0.000 \mathrm{I}, \mathrm{n}=5$ animals/group.

decreased MHC and costimulatory molecule expression. DCs are considered to be the main T cell stimulators due to their professional antigen-presenting function. ${ }^{23}$ And the function of $\mathrm{T}$ cell largely depends on cytokine pattern and expression of co-stimulatory molecules of DCs, and the Immature status of DCs could induce tolerance through induction of either $\mathrm{T}$ cell anergy, regulatory $\mathrm{T}$ cells, or production of regulatory cytokines. ${ }^{24,25}$ Our results also showed that inhibition of Dectin-1 could inhibit the antigen-presenting ability of DC, and inhibit the proliferation of $\mathrm{T}$ cells and the secretion of cytokines IL-17 and IFN- $\gamma$ in vitro.

To explore the effect of DC-Dectin-1-RNAi on the protection of islet grafts in mice, we selected day 7 and day 25 after transplantation to examine biological differences among the groups and changes in physiological function of grafts. Our results showed that DC-Dectin-1RNAi significantly prolonged the survival time of islet 
grafts, whereas DC-GFP exaggerated allograft rejection. Lu et $\mathrm{al}^{26}$ demonstrated that inhibiting the maturation of DCs could protect islet grafts, which is consistent with our finding. In addition, our results showed the glucose tolerance of DC-Dectin-1-RNAi treated recipients was better than that of the other groups, indicating prolonged survival of the islet allografts.

Though our results clearly showed that DC-Dectin1-RNAi prolonged the survival of islet allografts, the mechanism of this effect is unclear. Studies have shown that DCs can induce $\mathrm{T}$ cell expansion and Th cell subset polarization via the MHC and costimulatory molecules. ${ }^{27,28}$ Dectin-1 has positive effect on DC maturation, and inhibition of Dectin-1 inhibits the maturation of DCs. Study has shown that DCs with low expression of MHC and costimulatory molecules can induce antigen-specific tolerance. ${ }^{29}$ In the current study, DC-DC-Dectin-1-RNAi treated recipient mice exhibited an increase of Treg cells and reductions of Th1 and Th17 cells, and these changes were associated with alleviation of allograft rejection and prolonged allograft survival. INF- $\gamma$ and IL-17 inhibit the generation and function of Treg cells, and thus have a detrimental effect on transplant immunity. ${ }^{30}$ In addition, IL-10 was a well-known immunosuppressive cytokine that plays a key role in immune tolerance. ${ }^{31}$ Consistent with these prior studies, we observed a reduction of INF- $\gamma$ and IL-17 and increase of IL-10 levels in the DC-Dectin-1-RNAi group.

In this experiment, injection of DC-Dectin-1-RNAi prior to transplantation prolonged islet allograft survival; however, the allografts were eventually rejected. Thus, it remains to be explored if the persistent administration of DC-Dectin-1-RNAi will eventually induce immune tolerance. The exact mechanism by which Dectin-1 modulates DC function in a mouse islet transplant model also requires further study.

\section{Conclusion}

Inhibition of Dectin-1 prolonged islet allograft survival in mice, and was associated with decreased expression of MHC and costimulatory molecules on DCs, reduced numbers of Th1 and Th17 cells and associated cytokine levels, and an increase in the numbers of Treg cells. The results of this study suggested that Dectin-1 might have important therapeutic implications for new immunomodulating protocols in transplantation immunity.

\section{Funding}

This research was supported by grants from National Natural Science Foundation of China, China (81873591, and 81670591), Natural Science Foundation of Guangdong Province, China (2016A030311028), The Science and Technology Planning Project of Guangdong Province, China (2018A050506030), and The Science and Technology Program of Guangzhou, China (201704020073). The Guangdong Provincial Key Laboratory Construction Projection on Organ Donation and Transplant Immunology (2020B1212060026 and 2017B030314018), Guangdong Provincial International Cooperation Base of Science and Technology (Organ Transplantation) (2015B050501002).

\section{Disclosure}

The authors have declared no conflicts of interest for this work.

\section{References}

1. Lablanche S, Vantyghem MC, Kessler L, et al. Islet transplantation versus insulin therapy in patients with type 1 diabetes with severe hypoglycaemia or poorly controlled glycaemia after kidney transplantation (TRIMECO): a multicentre, randomised controlled trial. Lancet Diabetes Endocrinol. 2018;6:527-537. doi:10.1016/S22138587(18)30078-0

2. Rickels MR, Robertson RP. Pancreatic islet transplantation in humans: recent progress and future directions. Endocr Rev. 2019;40:631-668.

3. Rickels MR, Stock PG, de Koning EJP, et al. Defining outcomes for $\beta$-cell replacement therapy in the treatment of diabetes: a consensus report on the Igls criteria from the IPITA/EPITA opinion leaders workshop. Transpl Int. 2018;31:343-352. doi:10.1111/tri.13138

4. Xue D, Liu P, Chen W, Zhang C, Zhang L. An anti-CD103 antibodydrug conjugate prolongs the survival of pancreatic islet allografts in mice. Cell Death Dis. 2019;10(10):735. doi:10.1038/s41419-0191980-8

5. Ochando J, Ordikhani F, Jordan S, Boros P, Thomson AW. Tolerogenic dendritic cells in organ transplantation. Transpl Int. 2020;33(2):113-127. doi:10.1111/tri.13504

6. Obregon C, Kumar R, Pascual MA, Vassalli G, Golshayan D. Update on dendritic cell-induced immunological and clinical tolerance. Front Immunol. 2017;8:1514. doi:10.3389/fimmu.2017.01514

7. Brown GD, Crocker PR. Lectin receptors expressed on myeloid cells. Microbiol Spectr. 2016;4(5):10. doi:10.1128/microbiolspec.MCHD0036-2016

8. Takagawa T, Kitani A, Fuss I, et al. An increase in LRRK2 suppresses autophagy and enhances Dectin-1-induced immunity in a mouse model of colitis. Sci Transl Med. 2018;10(444):eaan8162. doi:10.1126/scitranslmed.aan8162

9. Yang AM, Inamine T, Hochrath K, et al. Intestinal fungi contribute to development of alcoholic liver disease. J Clin Invest. 2017;127 (7):2829-2841. doi:10.1172/JCI90562

10. Carvalho A, Giovannini G, De Luca A, et al. Dectin-1 isoforms contribute to distinct Th1/Th17 cell activation in mucosal candidiasis. Cell Mol Immunol. 2012;9(3):276-286. doi:10.1038/cmi.2012.1 
11. Zhao Y, Chu X, Chen J, et al. Dectin-1-activated dendritic cells trigger potent antitumour immunity through the induction of Th9 cells. Nat Commun. 2016;7:12368. doi:10.1038/ncomms12368

12. Kotthoff P, Heine A, Held SAE, Brossart P. Dexamethasone induced inhibition of Dectin-1 activation of antigen presenting cells is

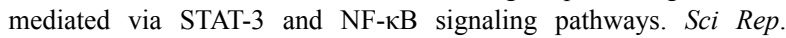
2017;7(1):4522. doi:10.1038/s41598-017-04558-z

13. Zorzi D, Phan T, Sequi M, et al. Impact of islet size on pancreatic islet transplantation and potential interventions to improve outcome. Cell Transplant. 2015;24(1):11-23. doi:10.3727/096368913X673469

14. Xue Z, Zhang X, Chen M, Lu X, Deng R, Ma Y. Dendritic cells transduced with single immunoglobulin IL-1-related receptor exhibit immature properties and prolong islet allograft survival. Front Immunol. 2017;8:1671. doi:10.3389/fimmu.2017.01671

15. Segura MM, Mangion M, Gaillet B, Garnier A. New developments in lentiviral vector design, production and purification. Expert Opin Biol Ther. 2013;13(7):987-1011. doi:10.1517/14712598.2013.779249

16. Gardner JK, Cornwall SMJ, Musk AW, et al. Elderly dendritic cells respond to LPS/IFN- $\gamma$ and CD40L stimulation despite incomplete maturation. PLoS One. 2018;13(4):e0195313. doi:10.1371/journal.pone.0195313

17. Tai A, Froelich S, Joo KI, Wang P. Production of lentiviral vectors with enhanced efficiency to target dendritic cells by attenuating mannosidase activity of mammalian cells. J Biol Eng. 2011;5(1):1. doi:10.1186/1754-1611-5-1

18. Zimara N, Chanyalew M, Aseffa A, et al. Dectin-1 positive dendritic cells expand after infection with leishmania major parasites and represent promising targets for vaccine development. Front Immunol. 2018;9:263. doi:10.3389/fimmu.2018.00263

19. Brown GD, Gordon S. Immune recognition. A new receptor for betaglucans. Nature. 2001;413(6851):36-37. doi:10.1038/35092620

20. Elder MJ, Webster SJ, Chee R, Williams DL, Hill Gaston JS, Goodall JC. $\beta$-glucan size controls dectin-1-mediated immune responses in human dendritic cells by regulating IL-1 $\beta$ production. Front Immunol. 2017;8:791. doi:10.3389/fimmu.2017.00791

21. Ito T, Hirose K, Norimoto A, et al. Dectin-1 plays an important role in house dust mite-induced allergic airway inflammation through the activation of CD11b+ dendritic cells. J Immunol. 2017;198(1):61-70. doi:10.4049/jimmunol.1502393

Journal of Inflammation Research

\section{Publish your work in this journal}

The Journal of Inflammation Research is an international, peerreviewed open-access journal that welcomes laboratory and clinica findings on the molecular basis, cell biology and pharmacology of inflammation including original research, reviews, symposium reports, hypothesis formation and commentaries on: acute/chronic inflammation; mediators of inflammation; cellular processes; molecular
22. Tian J, Zhang Y, Yang X, et al. Ficus carica polysaccharides promote the maturation and function of dendritic cells. Int J Mol Sci. 2014;15 (7):12469-12479. doi:10.3390/ijms150712469

23. Brossart P. Dendritic cells in vaccination therapies of malignant diseases. Transfus Apher Sci. 2002;27(2):183-186. doi:10.1016/ S1473-0502(02)00041-1

24. Moser M, Murphy KM. Dendritic cell regulation of TH1-TH2 development. Nat Immunol. 2000;1(3):199-205. doi:10.1038/79734

25 . Schülke $\mathrm{S}$. Induction of interleukin-10 producing dendritic cells as a tool to suppress allergen-specific $\mathrm{T}$ helper 2 responses. Front Immunol. 2018;9:455. doi:10.3389/fimmu.2018.00455

26. Lu X, Chen M, Xue Z, et al. Dendritic cells that highly express SOCS1 induce T-cell hypo-responsiveness and prolong islet allograft survival. Cell Immunol. 2017;314:36-41. doi:10.1016/j.cellimm.2017.01.011

27. Davidson MG, Alonso MN, Yuan R, et al. Th17 cells induce Th1polarizing monocyte-derived dendritic cells. J Immunol. 2013;191 (3):1175-1187. doi:10.4049/jimmunol.1203201

28. Kang JO, Lee JB, Chang J. Cholera toxin promotes Th17 cell differentiation by modulating expression of polarizing cytokines and the antigen-presenting potential of dendritic cells. PLoS One. 2016;11(6): e0157015. doi:10.1371/journal.pone.0157015

29. Wang Q, Liu Y, Wang J, et al. Induction of allospecific tolerance by immature dendritic cells genetically modified to express soluble TNF receptor. $J$ Immunol. 2006;177(4):2175-2185. doi:10.4049/ jimmunol.177.4.2175

30. Mohammadi F, Solgi G, Tajik M, et al. Enzyme-Linked Immunosorbent Spot (ELISpot) monitoring of cytokine-producing cells for the prediction of acute rejection in renal transplant patients. Eur Cytokine Netw. 2017;28(3):93-101. doi:10.1684/ecn.2017.0397

31. Shouval DS, Biswas A, Goettel JA, et al. Interleukin-10 receptor signaling in innate immune cells regulates mucosal immune tolerance and anti-inflammatory macrophage function. Immunity. 2014;40 (5):706-719. doi:10.1016/j.immuni.2014.03.011 